\title{
Aggregation-induced emissions of tetraphenylethene derivatives and their utilities as chemical vapor sensors and in organic light-emitting diodes
}

\author{
Yongqiang Dong, Jacky W. Y. Lam, Anjun Qin, Jianzhao Liu, \\ Zhen Li, and Ben Zhong Tang ${ }^{\text {a) }}$ \\ Department of Chemistry, The Hong Kong University of Science and Technology (HKUST), \\ Clear Water Bay, Kowloon, Hong Kong, China \\ Jiaxin Sun and Hoi Sing Kwok \\ Center for Display Research, HKUST, Clear Water Bay, Kowloon, Hong Kong, China
}

(Received 16 May 2007; accepted 5 June 2007; published online 5 July 2007)

\begin{abstract}
Nonemissive tetraphenylethene (TPE) 1 and its diphenylated derivative 2 were induced to emit intensely by aggregate formation. Crystalline aggregates of the dyes emitted bluer lights than their amorphous counterparts. The emissions of the TPE dyes could be switched off and on continuously and reversibly by wetting and dewetting with solvent vapors, respectively, manifesting their ability to optically sense volatile organic compounds. The light-emitting diodes fabricated from 1 and 2 were turned on at $\sim 2.9$ and $\sim 5 \mathrm{~V}$ and emitted blue lights with maximum luminance of $\sim 1800$ and $\sim 11000 \mathrm{~cd} / \mathrm{m}^{2}$, respectively. (C) 2007 American Institute of Physics. [DOI: 10.1063/1.2753723]
\end{abstract}

Development of efficient luminophoric materials has attracted much attention. ${ }^{1-4}$ Luminophoric dyes are usually used as solid films in the real-world applications such as organic light-emitting diodes (OLEDs). During the process of film formation, the dye molecules aggregate, which often results in a weakening in their emission intensities or even a total quenching of their light emissions. ${ }^{5-8}$ While much effort has been devoted to preventing chromophore aggregation, ${ }^{9-11}$ it is more desirable and useful to develop new luminophors that can emit efficiently in the aggregation state.

We have recently discovered an unusual phenomenon of aggregation-induced emission (AIE): a series of nonemissive molecules such as siloles, pyrans, fulvenes, and butadienes are induced to emit intensely by aggregate formation. ${ }^{12-16} \mathrm{~A}$ number of AIE molecules with different structures have subsequently been developed by various research groups. ${ }^{1-7,17-21}$ Many of these AIE dyes, however, are difficult to prepare, involving multistep reactions under stringent conditions. In this work, we synthesized TPE-based luminophors by a onestep reaction in a one-pot procedure and explored the possibility of utilizing the dyes as chemical sensors and OLED components.

TPE 1 and its diphenylated derivative 2 were readily prepared by simple McMurry reactions (Scheme 1). ${ }^{22}$ Almost no photoluminescence (PL) signals can be detected from their dilute solutions in acetonitrile and tetrahydrofuran (THF), revealing that the dyes are practically nonluminescent when molecularly dissolved in their good solvents. As shown in Fig. 1(a), the emission from the acetonitrile solution of 2 is so weak that its PL spectrum is virtually a flat line parallel to the abscissa. However, when a large amount of water $(>50 \%)$ is added into the solution, the PL of 2 is switched on. While the fluorescence quantum yield $\left(\Phi_{F}\right)$ of 2 in acetonitrile is $0.038 \%$, it is boosted to $50.3 \%$ when $90 \%$ water is added into acetonitrile [Fig. 1(b)]; noting that the

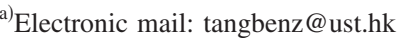

increase in $\Phi_{F}$ is a hefty 1324-fold. Similar effect was observed in the system of dye 1 .

Since water is a nonsolvent of the TPE dyes, the dye molecules thus must have aggregated in the water/ acetonitrile mixtures with high water contents. The aggregate formation is manifested by the level-off tails in the long wavelength region of the absorption spectra of 1 and 2 in the aqueous mixtures due to the Mie effect of the dye nanoparticles. Apparently the emissions of the TPE dyes are induced by aggregate formation; in other words, they are AIE active. In the dilute solutions, active rotations of the multiple phenyl rings against the ethylene core may have effectively nonradiatively deactivated the excited states, thus making the dyes nonemissive. Thanks to the steric effect of the peripheral phenyl rings, the dye molecules take propellerlike, nonplanar conformations (see crystal structure of 1 shown in Scheme $1)$, which prevents them from packing via $\pi$ - $\pi$ stacking interactions and quenching by such detrimental species as excimers. $^{22}$ The restriction of intramolecular rotations in the aggregates blocks the channel of nonradiative decay, hence changing the nonluminescent dyes to strong emitters.

Closer inspection of the PL spectra of 2 in the aqueous mixtures reveals that the emission maximum is redshifted when the water fraction is increased from $60 \%$ to $90 \%$. In the mixtures with "lower"water contents, the dye molecules may have clustered steadily to form ordered, bluer, crystalline aggregates. On the other hand, the dye molecules may have aggregated precipitately in the mixtures with "higher" water contents to form random, redder, amorphous aggregates.

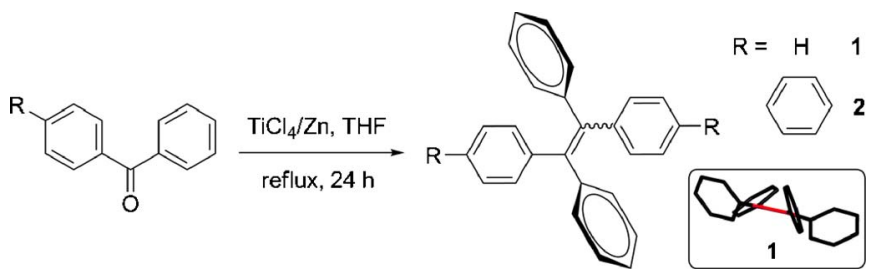

SCHEME 1. Syntheses of TPE dyes 1 and 2. Inset: crystal structure of 1 . 


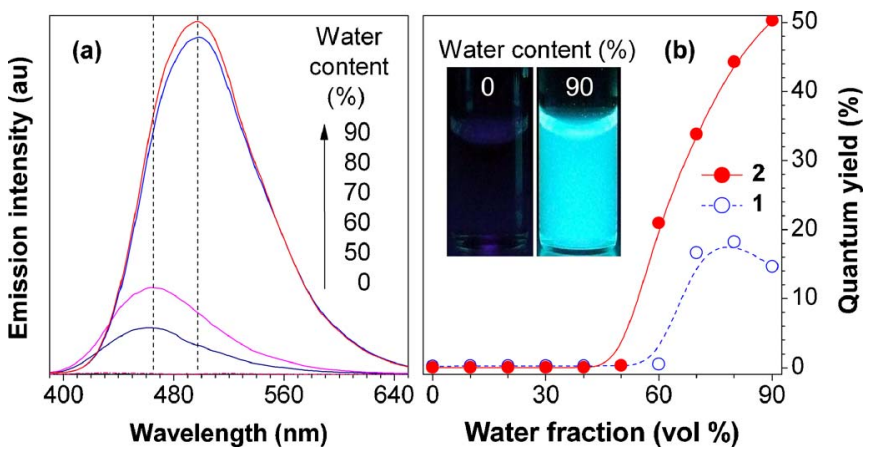

FIG. 1. (Color online) (a) PL spectra of $2(10 \mu \mathrm{M})$ in acetonitrile and water/acetonitrile mixtures $\left(\lambda_{\mathrm{ex}}=355 \mathrm{~nm}\right)$. (b) Plots of fluorescence quantum yields of 1 and 2 vs compositions of the water/acetonitrile mixtures. Inset: photos of the solutions of 2 in the water/acetonitrile mixtures with $0 \%$ and $90 \%$ water fractions.

Transmission electron microscope and electron diffraction analyses prove that the dye aggregates formed in the mixtures with "low" and "high" water contents are indeed crystalline and amorphous in nature, respectively.

We prepared crystals of 2 by recrystallization from an ethanol/THF mixture and its amorphous powders by rapidly quenching its isotropic melt with liquid nitrogen. The crystalline and amorphous natures of the samples were verified by x-ray diffraction analyses: the former gave many strong, sharp diffraction peaks at low angles, whereas the latter exhibited only weak, broad halos. Upon photoexcitation, the former and latter emit at 454 and $503 \mathrm{~nm}$, respectively [Fig. 2(b), inset]. This affirms that the crystalline and amorphous samples are indeed bluer and redder emitters, respectively, and demonstrates that the emission of the TPE dye can be tuned by changing its morphology.

The TPE dyes are nonemissive when dissolved in good solvents; we thus examined whether organic vapors can quench the emissions of their aggregates. As shown in Fig. 3, the emission from the spot of dye 2 on a thin-layer chromatography (TLC) plate (panel a) is turned off when the plate was put into a dish set saturated with vapors of chloroform (panel b). The emission becomes visible again when the vapors have been evacuated. The same effect is observed when other solvent (dichloromethane, THF, etc.) is used. Dye 1 behaves in a similar way. The solvent vapors may have condensed and formed thin liquid layers on the surfaces of the TLC plates, which have dissolved the absorbed dye mol-

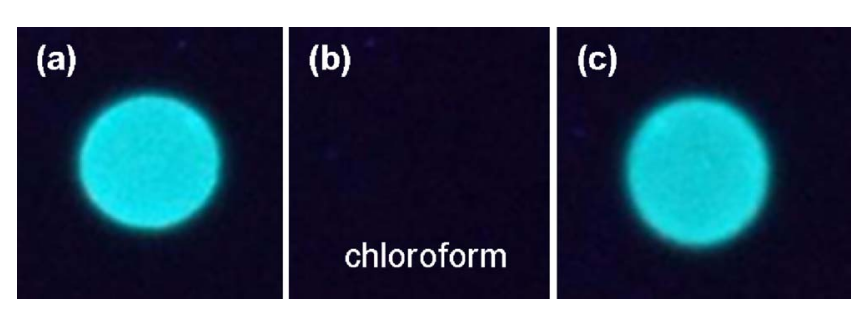

FIG. 3. (Color online) Photos of the spots of dye 2 on the TLC plates placed in the Petri-dish sets (a) without and (b) with saturated chloroform vapors. Photo in panel (c) was taken after the organic vapors in panel (b) had been evacuated.

ecules and subsequently quenched their light emissions. After vapor evacuation, the dye molecules aggregate and hence emit again. This off/on switching is fully reversible and repeatable for many times, because the involved process is a nondestructive physical cycle of dissolution-aggregation.

The AIE activity of the TPE dyes prompted us to study their OLED applications, for several AIE dyes we previously developed have shown outstanding device performances. ${ }^{23,24}$ We fabricated multilayer OLEDs with configurations of ITO/ NPB $(50 \mathrm{~nm}) / 1(30 \mathrm{~nm}) / \mathrm{BCP}(10 \mathrm{~nm}) / \mathrm{Alq}_{3}(20 \mathrm{~nm}) / \mathrm{LiF}$ $(1 \mathrm{~nm}) / \mathrm{Al} \quad(120 \mathrm{~nm})$ and ITO/NPB $(50 \mathrm{~nm}) / 2$ $(30 \mathrm{~nm}) / \mathrm{BCP} \quad(20 \mathrm{~nm}) / \mathrm{Alq}_{3} \quad(10 \mathrm{~nm}) / \mathrm{LiF} \quad(1 \mathrm{~nm}) / \mathrm{Al}$ $(150 \mathrm{~nm})$ by chemical-vapor deposition processes, in which $\mathrm{NPB}, \mathrm{BCP}$, and $\mathrm{Alq}_{3}$ were serving as hole-transport, holeblocking, and electron-transport layers, respectively. ${ }^{25}$

As shown in Fig. 4(a), the electroluminescence (EL) spectrum of 1 is peaked at $445 \mathrm{~nm}$ and nearly overlaps with the PL spectrum of its crystal, indicating that the emitting species in the device is the dye crystal. The OLED of 1 is turned on at a voltage as low as $\sim 2.9 \mathrm{~V}$, which is rare for a blue EL device. The OLED gives a maximum luminance of $\sim 1800 \mathrm{~cd} / \mathrm{m}^{2}$ at $\sim 7 \mathrm{~V}$ and a maximum external quantum yield of $\sim 0.4 \%$ at $4 \mathrm{~V}$. A maximum current efficiency of $0.45 \mathrm{~cd} / \mathrm{A}$ (equivalent to a power efficiency of $0.35 \mathrm{~lm} / \mathrm{W}$ ) is achieved under a current density of $\sim 55.6 \mathrm{~mA} / \mathrm{cm}^{2}$ at $\sim 4.0 \mathrm{~V}$.

Figure 2(a) shows the plots of current density and luminance of the OLED of 2 versus applied voltage. The device is turned on at $\sim 5 \mathrm{~V}$, with a maximum luminescence of $10680 \mathrm{~cd} / \mathrm{m}^{2}$ observed at $16 \mathrm{~V}$. It offers a maximum current efficiency of $5.15 \mathrm{~cd} / \mathrm{A}$ [Fig. 2(b)] and an external quantum yield of $2.56 \%$ at an applied voltage of $\sim 5.5 \mathrm{~V}$. The device
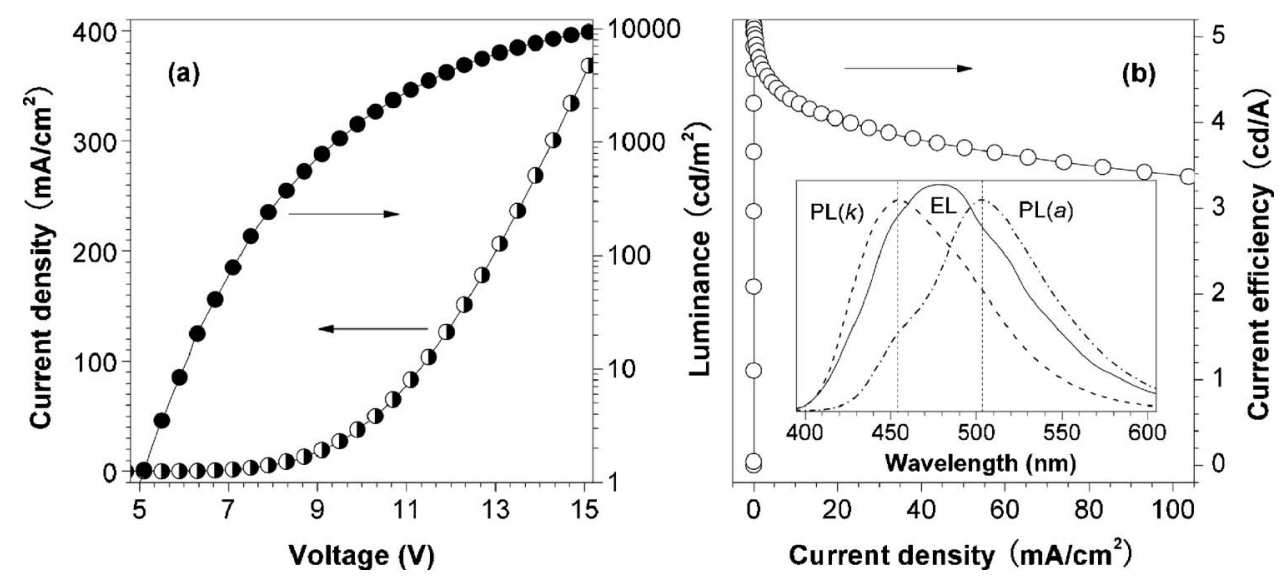

FIG. 2. (a) $I-V-L$ characteristic and (b) current efficiency of an OLED device of 2. Inset in panel (b): EL spectrum of the device and PL spectra of amorphous 

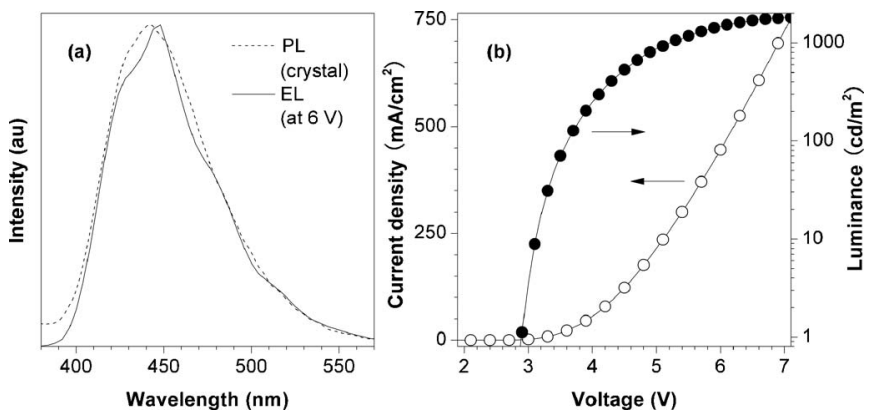

FIG. 4. (a) PL and EL spectra of dye 1 and (b) $I-V-L$ characteristic of its OLED.

of 2 shows an EL spectrum peaked at $476 \mathrm{~nm}$, locating between the PL spectra of its crystalline and amorphous samples. This indicates that the EL is from the partially crystallized dye molecules. The EL spectra of 1 and 2 do not change with increasing the applied voltage, revealing the excellent device stability originated from their AIE feature.

In summary, we have found that TPE dyes 1 and 2 are AIE active, which is rationalized to stem from the restriction of intramolecular rotations of their peripheral phenyl rings. We have proved that the blueshift in the PL of the dye aggregates is associated with a morphological transformation from amorphous to crystalline phase. The AIE effect of the TPE dyes has been utilized to sense chemical vapors and to enhance the performance and stability of their EL devices.

This work was partially supported by the Research Grants Council of Hong Kong (Project Nos. 602706, HKU2/ 05C, 603505 and 603304), the National Science Foundation of China (Project Nos. 50603021 and 20634020), and the Ministry of Science and Technology of China (Project No. 2002CB6130401).

${ }^{1}$ C. J. Bhongale and C.-S. Hsu, Angew. Chem., Int. Ed. 45, 1404 (2006). ${ }^{2}$ Q. Peng, Y. Yi, Z. Shuai, and J. Shao, J. Chem. Phys. 126, 114302 (2007). ${ }^{3}$ S. J. Toal, K. A. Jones, D. Magde, and W. C. Trogler, J. Am. Chem. Soc. 127, 11661 (2005).

${ }^{4}$ Y. Li, F. Li, H. Zhang, Z. Xie, W. Xie, H. Xu, B. Li, F. Shen, L. Ye, M.
Hanif, D. Ma, and Y. Ma, Chem. Commun. (Cambridge) 2007, 231.

${ }_{5}^{5}$ B.-K. An, S.-K. Kwon, S.-D. Jung, and S. Y. Park, J. Am. Chem. Soc. 124, 14410 (2002).

${ }^{6}$ S. Kim, Q. Zheng, G. S. He, D. J. Bharali, H. E. Pudavar, A. Baev, and P. N. Prasad, Adv. Funct. Mater. 16, 2317 (2006).

${ }^{7}$ Y. Liu, X. Tao, F. Wang, J. Shi, J. Sun, W. Yu, Y. Ren, D. Zou, and M. Jiang, J. Phys. Chem. C. 111, 6544 (2007).

${ }^{8}$ R. H. Friend, R. W. Gymer, A. B. Holmes, J. H. Burroughes, R. N. Marks, C. Taliani, D. D. C. Bradley, D. A. Dos Santos, J. L. Bredas, M. Logdlund, and W. R. Salaneck, Nature (London) 397, 121 (1999).

${ }^{9}$ S. H. Lee, B.-B. Jang, and Z. H. Kafafi, J. Am. Chem. Soc. 127, 9071 (2005).

${ }^{10}$ D. Marsitzky, R. Vestberg, P. Blainey, B. T. Tang, C. J. Hawker, and K. R. Carter, J. Am. Chem. Soc. 123, 6965 (2001).

${ }^{11}$ S. Setayesh, A. C. Grimsdale, T. Weil, V. Enkelmann, K. Muellen, F. Meghdadi, E. J. W. List, and G. Leising, J. Am. Chem. Soc. 123, 946 음 (2001).

${ }^{12}$ J. Luo, Z. Xie, J. W. Y. Lam, L. Cheng, H. Chen, C. Qiu, H. S. Kwok, X. Zhan, Y. Liu, D. Zhu, and B. Z. Tang, Chem. Commun. (Cambridge) 2001, 1740.

${ }^{13}$ J. Chen, C. C. W. Law, J. W. Y. Lam, Y. P. Dong, S. M. F. Lo, I. D. Williams, D. Zhu, and B. Z. Tang, Chem. Mater. 15, 1535 (2003).

${ }^{14}$ Y. Dong, J. W. Y. Lam, A. Qin, Z. Li, J. Sun, H. H. Y. Sung, I. D. Williams, and B. Z. Tang, Chem. Commun. (Cambridge) 2007, 40.

${ }^{15}$ Z. Li, Y. Dong, B. X. Mi, Y. H. Tang, M. Haussler, H. Tong, Y. P. Dong, J. W. Y. Lam, Y. Ren, H. H. Y. Sung, K. S. Wong, P. Gao, I. D. Williams, H. S. Kwok, and B. Z. Tang, J. Phys. Chem. B 109, 10061 (2005).

${ }^{16}$ G. Yu, S. Yin, Y. Liu, J. Chen, X. Xu, X. Sun, D. Ma, X. Zhan, Q. Peng, Z. Shuai, B. Z. Tang, D. Zhu, W. Fang, and Y. Luo, J. Am. Chem. Soc. 127, 6335 (2005).

${ }^{17}$ J.-M. Kim, Macromol. Rapid Commun. 28, 1191 (2007).

${ }^{18}$ M. R. Han, Y. Hirayama, and M. Hara, Chem. Mater. 18, 2784 (2006).

${ }^{19}$ Z. Wang, H. Shao, J. Ye, L. Tang, and P. Lu, J. Phys. Chem. B 109, 19627 (2005).

${ }^{20}$ Z. Xie, B. Yang, W. Xie, L. Liu, F. Shen, H. Wang, X. Yang, Z. Wang, Y. Li, M. Hanif, G. Yang, L. Ye, and Y. Ma, J. Phys. Chem. B 110, 20993 (2006).

${ }^{21}$ Y. Qian, S. Li, G. Zhang, Q. Wang, S. Wang, H. Xu, C. Li, Y. Li, and G. Yang, J. Phys. Chem. B 111, 5861 (2007).

${ }^{22}$ H. Tong, Y. Hong, Y. Dong, M. Haussler, J. W. Y. Lam, Z. Li, Z. Guo, Z. H. Guo, and B. Z. Tang, Chem. Commun. (Cambridge) 2006, 3705.

${ }^{23}$ H. Chen, W. Y. Lam, J. Luo, Y. Ho, B. Z. Tang, D. Zhu, M. Wong, and H. S. Kwok, Appl. Phys. Lett. 81, 574 (2002).

${ }^{24}$ H. Chen, J. Chen, C. Qiu, B. Z. Tang, M. Wong, and H.-S. Kwok, IEEE J. Sel. Top. Quantum Electron. 10, 10 (2004).

${ }^{25} \mathrm{Al}_{3}=$ tris (8-hydroxyquinolinolato)aluminum, $\quad \mathrm{BCP}=2,9$-dimethyl-4, 7-diphenyl-1,10-phenanthroline, ITO=indium tin oxide, $\mathrm{NPB}=N, N^{\prime}$-di (1-naphthyl)- $N, N^{\prime}$-diphenylbenzidine. 